\title{
Racial/Ethnic Heterogeneity and Rural-Urban Disparity of COVID-19 Case Fatality Ratio in the USA: a Negative Binomial and GIS-Based Analysis
}

\author{
Ayodeji E. lyanda $^{1}$ (D) Kwadwo A. Boakye ${ }^{2}$ (D) Yongmei Lu $^{1}$ (D) Joseph R. Oppong ${ }^{3}$ (D)
}

Received: 31 December 2020 / Revised: 1 February 2021 / Accepted: 21 February 2021 / Published online: 26 February 2021

(C) W. Montague Cobb-NMA Health Institute 2021

\begin{abstract}
The 2019 coronavirus disease (COVID-19) has exacerbated inequality in the United States of America (USA). Black, indigenous, and people of color (BIPOC) are disproportionately affected by the pandemic. This study examines determinants of COVID-19 case fatality ratio (CFR) based on publicly sourced data from January 1 to December 18,2020 , and sociodemographic and rural-urban continuum data from the US Census Bureau. Nonspatial negative binomial Poisson regression and geographically weighted Poisson regression were applied to estimate the global and local relationships between the CFR and predictors - rural-urban continuum, political inclination, and race/ethnicity in 2407 rural counties. The mean COVID-19 CFR among rural counties was 1.79 (standard deviation $(\mathrm{SD})=1.07 ; 95 \%$ CI $1.73-1.84$ ) higher than the total US counties $(M=1.69$, $\mathrm{SD}=1.18 ; 95 \% \mathrm{CI}: 1.65-1.73)$. Based on the global NB model, CFR was positively associated with counties classified as "completely rural" (incidence rate ratio $(\mathrm{IRR})=1.24 ; 95 \% \mathrm{CI}: 1.12-1.39)$ and "mostly rural" (IRR $=1.26 ; 95 \%$ CI: $1.15-1.38$ ) relative to "mostly urban" counties. Nonspatial regression indicates that COVID-19 CFR increases by a factor of 8.62, 5.87, 2.61, and 1.36 for one unit increase in county-level percent Blacks, Hispanics, American Indians, and Asian/Pacific Islanders, respectively. Local spatial regression shows CFR was significantly higher in rural counties with a higher share of BIPOC in the Northeast and Midwest regions, and political inclination predicted COVID-19 CFR in rural counties in the Midwest region. In conclusion, spatial and racial/ethnic disparities exist for COVID-19 CFR across the US rural counties, and findings from this study have implications for public health.
\end{abstract}

Keywords Rural-urban continuum - COVID-19 severity · Political inclination · Racial/ethnic minorities · Disease severity · Spatial analysis

\section{Introduction}

Although abundant medical and scientific reports were published following the previous severe acute respiratory syndrome coronavirus (SARS-CoV) outbreaks in 2003/2004 [1] and the 2019 novel coronavirus disease (COVID-19), there is a lack of up-to-date study on the rural-urban continuum and

Ayodeji E. Iyanda

aei11@txstate.edu

1 Department of Geography, Texas State University, San Marcos, TX, USA

2 School of Biological and Population Health Sciences, Oregon State University, Corvallis, OR, USA

3 Department of Geography and the Environment, University of North Texas, Denton, TX, USA racial disparity of case fatality ratio (CFR) in the United States of America (USA). Furthermore, a comprehensive empirical study on determinant factors predicting the local disparity of CFR for different geographic units in the USA is presently scarce. This study defines CFR as the ratio of deaths from COVID-19 and confirmed total COVID-19 cases in 2020 to indicate disease severity $[2,3]$. Hard outcomes such as the CFR are critical not only for determining the severity of a disease but also for formulating intervention strategies and policies from a public health perspective. It is important to note that CFR is different from the mortality rate. ${ }^{1}$ While mortality rate reflects the total number of deaths scaled to the size of population, not everyone is equally exposed to the disease $[4,5]$. CFR, however, measures the severity of a

\footnotetext{
${ }^{1}$ Mortality rate is calculated based on the total population in a geographic area, such as a county, state, or a country. Hence, CFR is determined based on those who were tested and confirmed positive. The related issue has been discussed by Spychalski et al. [4].
} 
disease, an essential aspect of the health outcomes from medical treatment and health management of COVID-19 cases. Spatial analysis of CFR is essential to understand the geographic disparity and potential underlying factors that might contribute to the spatial patterns of health outcomes of COVID-19 cases, particularly in rural counties.

The USA presently has the highest officially reported cases and death from COVID-19 [6], and COVID-19 and the US COVID-19 index case was reported on January 20, 2020, while the first coronavirus death was reported on February 29, 2020 [7]. Since the recent outbreak of COVID-19 in China, the virus has diffused to over 210 countries and territories, infecting over 75 million people with 1,699,039 deaths as of December 20, 2020, making it one of the deadliest the world has ever seen $[8,9]$. In the USA, COVID-19 had infected over 19 million and killed over 330,000 people by Christmas day, 2020. Like other deadly infectious diseases, eradicating COVID-19 is extremely difficult considering the human population as its possible host [1]. As of writing this paper, a few antiviral vaccines have been successfully developed and approved, but the distribution and the longtime efficiencies are not readily determined. As of December 20, 2020, the daily US CFR was $1.78 \%$, lower than the global rate $(2.2 \%)$ but higher than CFR in Germany (1.5\%) and India (1.5\%) [10]. However, there is a stark variation of CFR within the country - at different administrative regions (e.g., states, counties, cities, rural-urban). This study examines the geographic variations of COVID-19 CFR, prioritizing US rural counties.

Previous COVID-19 studies have shown that COVID-19 disproportionately affects rural communities with a high percentage of minority racial/ethnic groups [11-14]. Following recent studies that focus on the disparity in COVID-19 burden by race and ethnicity in rural areas, this paper examined racial heterogeneity in COVID-19 CFR in the USA. We used data from public sources between January 1 and December 18, 2020 , to unpack the current evidence of a racial and geographic disparity as well as political inclination on the fatality ratio in the USA. There are several reasons to consider health disparity in rural settings during a pandemic, including scarce resources for resilience and recovery. Rural communities generally have low access to health care due to the low population density that can sustain the most needed health care resources (e.g., doctors, specialist health facilities) [15].

\section{Determinants of COVID-19 Disparity}

Fatality arising from COVID-19 in the USA is determined by several contextual (rural/intermediate/urban, college town/ noncollege town, presence of institutions such as prisons, nursing homes) [13] and compositional (racial composition, age, sex) factors. This pattern has been explained using the social determinants of health $(\mathrm{SDOH})$ framework in several studies, including research on COVID-19 [16-18]. The urban areas are often the focus of many studies and public health interventions. Although the virus incidence was highest in the dense and populated urban centers (e.g., New York, Los Angeles-California), the epidemic has also spread outside of big cities [19] with possibly important implications for the evolution of the reproduction number $\left(R_{\mathrm{t}}\right)$ and increased mortality in rural areas.

Substantial evidence on the role of SDOH in understanding COVID-19 health disparity has been documented in the literature. Seligman et al. [20] linked data from the National Health and Nutrition Examination Survey (NHANES) and publicly reported data on COVID-19 deaths from the public health agencies of the USA, China, the UK, Spain, Italy, and France to examine the role of SDOH on COVID-19 deaths among randomly sample individuals from the NHANES. They analyzed the distributions of COVID-19 deaths by race/ethnicity, income, education level, and veteran status. Their results show large social gradients in COVID-19 mortality. Notably, adults from households earning less than the median income made up two-thirds $(67.7 \%)$ of COVID-19 deaths, while those with less than a high school education accounted for approximately 1 in 4 deaths. Compared to White, a higher magnitude of death $(54.8 \%)$ occurred among nonWhite races/ethnicities [20].

The current geographic disparities in COVID-19related deaths and infection rates can, to a large extent, be related to the variation in the non-pharmaceutical interventions (NPIs) adopted by the different state and local governments [21]. Examples of NPIs include stay-athome, social distancing, institutional lockdown (e.g., schools), ban of domestic and international travel, voluntary isolation, and compulsory suspected case quarantine. These measures differ across different regions and equally reinforce variances in the epidemiological outcomes (e.g., attack and mortality rates). The degree of implementation of various non-pharmaceutical measures and the level of effective reproduction number $\left(R_{\mathrm{t}}\right)$ at the time of the intervention determine the corresponding suppression rate, that is, the average number of people each infected person can spread the virus to. New evidence of NPIs indicates a significant aversion in the supposed death rate, depending on the responsiveness of the government of a country, a state, or a county. In the USA, evidence of early implementation of non-pharmaceutical measures was recorded in California as opposed to places like New York, New Jersey, and Massachusetts.

Since the disease outbreak, several attempts have been made to investigate factors driving the variability of COVID-19 infection, mortality rate, and CFR at different geographic scales using different approaches [11, 21-25]. A 
multicountry study shows that the political regime, economic parameters, chronic health conditions, behavioral factors (e.g., smoking), and demography are associated with CFR variation among countries [22]. Similarly, Khan et al. [25] used negative binomial regression to determine country-level case fatality rate from COVID-19 in 86 countries. They reported that higher health care capacity was related to lesser case fatality with every additional unit increase in the health care capacity index associated with a $42 \%$ decrease in the case fatality. Perone [26] used ordinary least squares regression in Italy and found that the aging population, car and firm density, air pollutant concentrations, relative average humidity, COVID19 prevalence, and health system saturation were significant drivers of CFR. In New York state, Ge, Sun, and Shen [26] performed subgroup analysis among patients with hypertension and diabetes by age and calculated the CFR and relative ratio (RR) for ages of 18-44, 45-64, and 65+ years using simple linear regression. The authors found that the older population had a higher CFR, but the elevated RRs associated with comorbidities are more pronounced among the younger population. However, as far as we know, none of these studies considered rural-urban disparity and racial/ethnic heterogeneity in the analysis of COVID-19 CFR. Specifically, a limited study in the USA has examined the spatial variation of COVID-19 CFR and their determinants. Hence, there is a need for a preliminary study to show the geographic distribution of CFR in the USA, particularly in nonmetropolitan areas.

Using geospatial methods, Anderson et al. [24] analyzed the spatial determinants of local COVID-19 transmission among US counties. Based on cluster analysis, COVID-19 hotspots were predominant in New England and the US's Southeast and Southwest regions. Spatial lag regression showed that living in urban areas, micropolitan areas, or small towns and a higher proportion of Black individuals and nonEnglish speakers were significant predictors of spatial patterns of COVID-19 cases. At the same time, a higher proportion of Black individuals and persons living with a disability were significant predictors of COVID-19 infection [24]. To test for the validity of the spatial lag, the authors performed a mixed-effect model. They observed that significant predictors of deaths and cases in the county-level spatial lag model remained significant at the state level despite variability in social distancing behaviors, stay-at-home mandates, and funding for assistance programs such as Medicaid. Sun et al. [23] analyzed the geographic distribution and determinants of COVID-19 period prevalence and showed that the countylevel racial/ethnic composition is important in determining the period prevalence. Notably, percent Black and Hispanics had the highest contribution relative to percent White.

Possible ways the pandemic is linked to the political milieu have been explained in a recent study [14]. Central to the 2020 election campaign was how to control and possibly eradicate the pandemic in the USA. During the election campaign by different political parties across the country, different NPI implementation strategies and public health protocols were adopted, including wearing face masks. However, not all parties adhere to face masks' use, as recommended by the Centers for Disease and Prevention (CDC) during political meetings [27]. Furthermore, to reduce the infection rate during the last US elections, voting by mail was largely supported by the Democratic party, while the Republican party preferred inperson voting due to the fear of vote suppression [28]. It turned out that more people opted for mail by ballot than ever recorded in the history of the American election. Though mail by ballot is not new, the outgoing administration was very critical of it.

High population density is one of the risk factors of disease transmission in urban areas because such places usually exhibit high socio-spatial and economic interactions. Unfortunately, earlier health geographical evidence on earlier pandemics' evolution has not fully supported this assertion $[29,30]$. An ecological study examined the spatial disparities in COVID-19 cases and deaths using several predictors, including population density in 2814 US counties [19]. The study argues that high population density not necessarily indicates a disease hotspot. For instance, the study found that although Detroit and New Orleans metropolitan areas were ranked lower in terms of population, they had one of the largest hotspots. Los Angeles and Chicago, on the other hand, were ranked as the second and third largest metropolitan areas, respectively, in the study unexpectedly ranked lower in terms of infections and deaths relative to their population rankings [19]. However, regression analysis confirmed that population density was a significant determinant of infections and death across counties in the USA. Meanwhile, when they subjected their analysis to a sensitivity test, population density became an insignificant predictor for infections, and the model explained less than $1 \%$ of the variations of infections in rural data alone. The authors concluded that population density is less likely to be a risk factor for rural counties because rural areas generally have much lower population densities.

While rural communities in the USA are predominantly White, evidence has shown that the minority racial/ethnic groups suffer the worst health outcomes [31, 32]. For example, using national survey data from 1936 to 2016, Singh et al. [16] found that life expectancy (LE) in the USA increased substantially among the general population, but it was lowest among African Americans. The Black infant mortality rate was 2.3 times higher than in White infants, and infant/child mortality was exceptionally higher in rural areas and poor communities. Among the adult population, the study also showed that racial/ethnic, socioeconomic, and geographic disparities manifested in mortality and morbidity from cardiovascular disease, cancer, diabetes, COPD, HIV/AIDS, homicide, psychological distress, hypertension, smoking, obesity, and access to quality health care [16]. Evidence in the USA has also shown that the disparity gap widens over time. In an 
earlier analysis of rural-urban disparity in LE, researchers noted that rural poor and rural Blacks experienced survival probabilities that urban rich and urban Whites enjoyed more than four decades earlier [33].

A racial disparity is also more exacerbated by COVID19. According to a recent report, COVID-19 decreased the LE in the USA by one full year by the middle of 2020 of which it was greater for non-Hispanic Black and Hispanic populations [34]. For example, the gap in LE between non-Hispanic white and Black people widened from 4.1 years in 2019 to six years in the first half of 2020. The report indicates that LE at birth for the total U.S. population declined from 78.8 years in 2019 to 77.8 years for January through June 2020. During that same time period, LE for non-Hispanic Black people declined by 2.7 years (74.7 to 72), 1.9 years for Hispanic individuals (81.8 to 79.9 ), and by 0.8 years for non-Hispanic white people (78.8 to 78 ).

In a recent study, Peters [12] reported that about onethird of rural counties in the USA are highly susceptible to COVID-19. The author operationalized susceptibility as the proportion of older and health-compromised populations and care facilities for the elderly. The author defined high rural vulnerabilities based on the fewer physicians, lack of mental health services, higher disability, and proportion of uninsured poor minority rural residents. $\mathrm{He}$ further emphasized that poor Internet access limits telemedicine, and encroachment of social capital and social services may hinder the local pandemic recovery rate in vulnerable communities. Environmental justice literature also found that Hispanics/Latino communities experience highly segregated communities and are likely to be exposed to environmental insults, in addition to the current disparity in COVID-19 death and hospitalization [35-38].

Given the background, it is crucial to investigate the rural-urban continuum variation of COVID-19 CFR to inform socio-health policies and reduce inequality. Based on a priori knowledge, it is hypothesized that CFR will vary geographically depending on the heterogeneous compositional and contextual characteristics of counties. We also hypothesized that political inclination would impact the variation in COVID-19 CFR. However, the rate at which data on COVID-19 is unfolding makes epidemiological study a daunting task at the moment. As a result, we used 354 days of data between January 1 and December 18, 2020, to cover a whole pandemic year. This study has three specific research objectives. First, it investigates the COVID-19 case fatality ratio's distribution and predictors based on the ruralurban continuum. Second, it examines the effect of racial composition in the distribution of COVID-19 case fatality ratio in rural counties only. Lastly, it examines the probable effect of political inclination in the predictions of COVID19 case fatality ratio.

\section{Methods}

The present study is a cross-sectional examination of the COVID-19 case fatality ratio (CFR) in the USA, and the unit of analysis is the county level. The study is a conditional estimation of COVID-19 CFR based on the confirmed cases and death from COVID-19 from January 1 to December 18, 2020 .

\section{Data and Measures}

Data for this study were from publicly available sources: the New York Times and US Census Bureau. COVID-19 data are drawn from the New York Times database [39], updated daily with reports from public health authorities around the country. The New York Times compiles time-series data from state and local governments and health departments to provide a complete record of the ongoing outbreak. Since January, the Times has tracked cases of coronavirus in real time as they were identified after testing. The data reported at the county level used here include the number of new confirmed cases and deaths each day in each county. The total infection of COVID-19 in all the US counties, including Puerto Rico, from January 1 to December 18,2020, was 17,451,388, with a total death of 311,812 being reported.

\section{Dependent Variable}

The dependent variable, case fatality ratio (CFR), was calculated based on the World Health Organization's [3] definition of CFR as the proportion of individuals diagnosed with COVID-19 who died from the disease (Eq. 1).

Case fatality ratio, CFR

$$
=\frac{\text { number of deaths from COVID-19 }}{\text { number of confirmed cases of COVID-19 }} \times 100
$$

\section{Independent Variables}

Rurality, the main independent variable, was determined based on the 2010 Decennial Census; the Bureau reported a proportion of each county's population that lived in a rural area [13]. The determination of the rural-urban continuum follows the US Bureau's categorization of counties: (1) completely rural (100\%), (2) mostly rural (50-99.9\%), and (3) mostly urban ( $\leq 50 \%$ rural). According to another published work that used the data [13], counties with $50 \%$ or more rural population are referred to as "rural." Race/ethnic composition was the second independent variable. All county population data, including race/ethnicity composition, are 2018 estimates from the US Census Bureau [40]. 


\section{Covariates}

Other covariates include the county population density calculated as residents per square mile, using the 2019 Census Bureau's land area estimates. Note that two counties, Oglala Lakota, SD, and the Kusilvak Census Area, AK, are not included in the Census file, but their land areas were retrieved from Wikipedia. A master file and the code for generating and compiling the data are available from [41]. Data on the 2020 Presidential Election at the county-level were retrieved from Politico [42].

\section{Data Analysis}

Univariate analysis was used to present the descriptive analysis, and analysis of variance was used to determine the ruralurban variation of CFR. Because of the dependent variable examined here, the case fatality ratio follows a Poisson distribution, and a negative binomial (NB) Poisson model was preferred to assess the relationship between the standardized ratio presented in Eq. 1 and some selected predictors following previous studies that have applied Poisson models to estimate disease rates such as mortality rates from diseases [43, 44]. The predictors were selected based on the existing rural health literature, particularly from recent studies on the COVID-19 disparity in the USA $[29,30,35]$. In the first analysis, the primary independent variable was the rural-urban continuum described above. Political inclination was operationalized as the percentage of votes at the county level for the two US 2020 presidential candidates (Joe R. Biden of the Democratic party (reference) vs. President Donald J. Trump of the Republican party) to determine the disparity in the rural-urban case fatality ratio. Three models were developed. Model 1 included only the rural-urban categorical variable, and the areas categorized as mostly urban were used as a reference category. Model 2 included all the variables in model 1 plus the political inclination variable. Model 3 included all the variables in model 2 plus racial/ethnic compositions (percent non-Hispanic Black alone, percent Hispanics, percent American Indian alone, percent Asian/Pacific Island), county-level household median income, and population density. In addition, we conducted a sensitivity analysis to show the impact of the 2-week increase in cases and deaths from COVID-19 on the variation of county-level CFR of COVID-19.

To examine the racial disparity in COVID-19 case fatality ratio in rural areas, we subset our data to counties classified as rural (mostly or completely rural, 50-100). Model 1 included racial/ethnic composition as the primary independent variable. Model 2 included all variables plus political inclination while

\footnotetext{
${ }^{2} Z$-scores are expressed in terms of standard deviations from their means. They have a distribution with a mean of 0 and a standard deviation of 1 .
}

controlling for household income and population density. The standardized scores ${ }^{2}$ of household income, population density, racial/ethnic composition, and political inclination variables were used for straightforward comparison. We reported the model effects using the incidence rate ratio (IRR) and their associated $95 \%$ confidence intervals (CI). All nonspatial analyses were conducted in SPSS version 20 [45].

\section{Spatial Analysis of Rural Case Fatality Ratio}

In addition to the standard negative binomial Poison regression, we model COVID-19 case fatality ratio in US rural counties using spatial econometrics techniques [46]. Nonparametric geographically weighted regression (NPGWR) was used because of the Poisson distribution of the outcome variable. In the standardized scores of Black, indigenous, and people of color (BIPOC) and based on a recent commentary published in Lancet [14], the percent share of Trump win in 2020 is used to represent the impact of political inclination on COVID-19 case fatality ratio. To address our hypothesis that the political inclination partly influences the racial/ethnic inequality in COVID-19 severity in the USA [14], we used the county-level percent minority racial/ ethnic groups (i.e., BIPOC) and the percent share of Trump voters. For this aspect of the analysis, we considered only rural counties $(n=2407)$. We used GWR to determine the nonstationarity in the relationship between the predictors and COVID-19 CFR. We specified bisquare kernel as the local weighting scheme to determine the weight of one feature in a location, $w_{\mathrm{i}}$, and weights for the surrounding feature in another location, $w_{\mathrm{j}}$. Optimal bandwidth for modeling the predictors and "Golden search" was used to determine the maximum neighborhoods needed or optimal bandwidth to determine location statistics based on the lowest value of corrected Akaike Information Criterion (AICc). Unlike the traditional regression, GWR estimates a unique regression model for each geographic unit instead of assuming a global fit for all the 2407 counties. Besides, hotspot analysis was conducted using the local Getis-Ord Gi* technique using ArcGIS Pro [47].

\section{Results}

\section{Descriptive Results: All Counties}

The mean of COVID-19 CFR ranged from a low 1.56 in counties categorized as mostly urban to 1.79 in mostly rural counties (Table 1). Nonparametric analysis of variance confirmed that CFR significantly varies by rural-urban continuum (Chi-square $\left._{(2)}=33.69, p<0.001\right)$. Figure 1 shows that the median difference of COVID-19 CFR in urban counties was 
Table 1 Descriptive statistics of COVID-19 case fatality ratio by rural-urban continuum

\begin{tabular}{lcccc}
\hline Variable & Mean & Standard error of mean & Std. deviation & Min-max \\
\hline Mostly urban & 1.561 & 0.029 & 1.061 & $0.000-7.692$ \\
Completely rural & 1.779 & 0.057 & 1.520 & $0.000-10.000$ \\
Mostly rural & 1.792 & 0.031 & 1.071 & $0.000-8.333$ \\
Rural only & 1.787 & 0.029 & 1.257 & $0.000-10.000$ \\
Total (USA) & 1.694 & 0.021 & 1.185 & $0.000-10.000$ \\
\hline
\end{tabular}

not significantly different from counties categorized as completely rural (standardized test statistics $=-1.53$, adj. $p$ $>0.05$ ) but significantly different between mostly urban and mostly rural counties (standardized test statistics $=-5.74$, adj. $p<0.001)$. Also, the CFR significantly differs between the two categories of rurality (standardized test statistics $=-3.31$, adj. $p=0.003)$.

\section{Multivariate Results: All Counties}

Table 2 presents the result of the standard NB regression. In models 1 and 2 , the range of CFR incidence rate ratio in counties categorized as mostly rural or completely rural was $15-26 \%$ and $15-24 \%$, respectively. Political inclination measured as the outcome of the US presidential election reduced the rurality effect in model 2 . Counties where the Republican

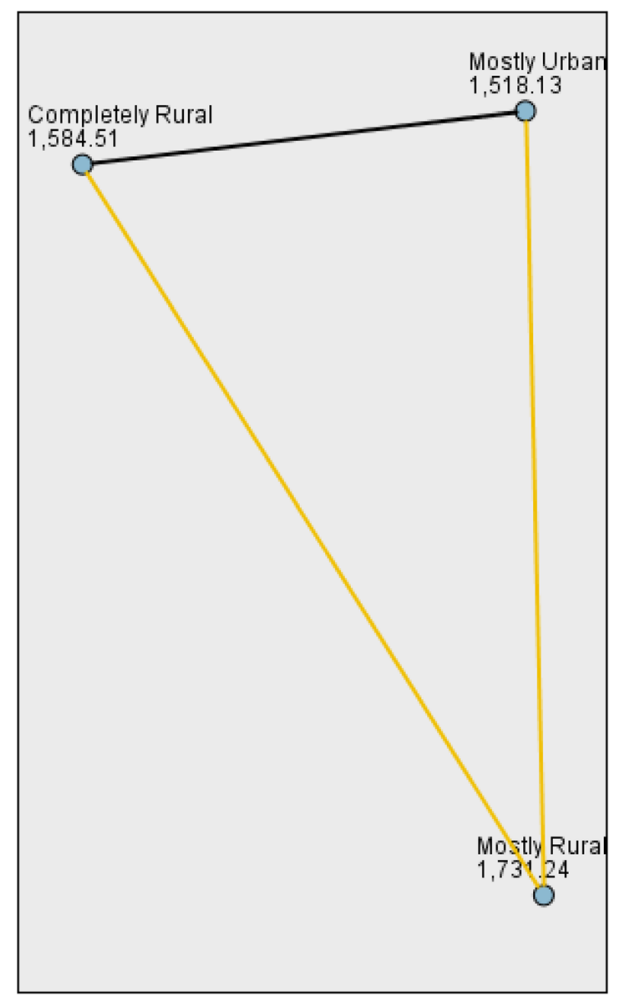

Fig. 1 Pairwise comparisons of COVID-19 case fatality ratio by ruralurban continuum (note: each node shows the sample average rank of the rural-urban continuum) party candidate had major voters predicted a $9 \%$ decrease in COVID-19 CFR (IRR= 0.91, 95\% CI: 0.83-0.99), compared to counties where the Democratic presidential candidate had major voters. We note that the incidence rate ratio of rurality was attenuated in model 3 after introducing other covariates. Specifically, the magnitude of the incidence rate ratio of counties classified as mostly rural was reduced to $18.4 \%$ from $26 \%$ in model 1 , and the incidence rate ratio of completely rural significantly remained unchanged. Holding other factors constant, CFR was negatively associated with median household income. Though the association did not reach statistical significance, CFR was positively associated with population density. Notably, all racial/ethnic categories exhibit a positive association with CFR in the full model (Table 2). To better visualize the racial/ethnic disparity of COVID-19 CFR, we present the incident rate ratio in Fig. 2. County-level percent Black and percent Hispanics composition had the highest incident rate ratio.

\section{Rural County Racial Composition and COVID-19 CFR}

Figure 3 presents the COVID-19 CFR in the US rural counties alone, which shows significant hotspots and coldspots of COVID-19 CFR, mostly in the Northwest, Northeast, and Southeast regions. Focusing only on rural counties in Table 3, the association between the percentage of the four minority racial/ethnic groups and the COVID-19 CFR was positive and significant. Regression indicates that for every one unit increase on the rural county-level percent BIPOC, CFR increases by a factor of 8.62, 5.87, 2.61, and 1.36 for the county-level percentage of Blacks, Hispanics, American Indian, and Asian/Pacific Islander, respectively (Table 3). Figure 4 shows that after adjusting for other covariates, the pattern of COVID-19 CFR remained unchanged for the percentage of Blacks in rural communities, but it changed positively for the percentage of American Indians and percent Hispanics, and reduced for the percentage of Asian/Pacific Islander. Again, the political inclination was not related to the pattern of COVID-19 CFR for racial/ethnic minority groups in the model for rural counties alone. In addition to the separate models presented above, we run a sensitivity analysis to examine the impact of other covariates' impacts on the variation of COVID-19 CFR using all the data. Table 4 
Table 2 Models for predicting COVID-19 case fatality ratio in all the US counties

\begin{tabular}{|c|c|c|c|c|c|c|c|c|c|}
\hline \multirow[t]{3}{*}{ Parameter } & \multicolumn{3}{|c|}{ Model 1} & \multicolumn{3}{|c|}{ Model 2} & \multicolumn{3}{|c|}{ Model 3} \\
\hline & \multirow[t]{2}{*}{ IRR } & \multicolumn{2}{|c|}{$95 \% \mathrm{CI}$} & \multirow[t]{2}{*}{ IRR } & \multicolumn{2}{|l|}{$95 \% \mathrm{CI}$} & \multirow[t]{2}{*}{ IRR } & \multicolumn{2}{|c|}{$95 \% \mathrm{CI} \exp (\mathrm{B})$} \\
\hline & & Lower & Upper & & Lower & Upper & & Lower & Upper \\
\hline (Intercept) & 4.77 & 4.47 & 5.08 & $5.29^{\ddagger}$ & 4.95 & 5.66 & 5.11 & 4.77 & 5.48 \\
\hline \multicolumn{10}{|c|}{ Rural-urban continuum (mostly urban ${ }^{\text {ref. }}$ ) } \\
\hline Mostly rural & 1.26 & 1.15 & 1.38 & 1.15 & 1.04 & 1.27 & 1.18 & 1.07 & 1.31 \\
\hline Completely rural & 1.24 & 1.12 & 1.39 & 1.15 & 1.03 & 1.29 & 1.24 & 1.11 & 1.40 \\
\hline Trump vote (Biden vote ${ }^{\text {ref. }}$ ) & & & & 0.91 & 0.83 & 0.99 & 1.05 & 0.96 & 1.15 \\
\hline Median household income & & & & & & & 0.88 & 0.83 & 0.92 \\
\hline Population density & & & & & & & 1.05 & 0.99 & 1.10 \\
\hline \multicolumn{10}{|l|}{ Racial composition (White ${ }^{\text {ref. }}$ ) } \\
\hline Percent Black & & & & & & & 8.33 & 4.90 & 14.17 \\
\hline Percent Hispanics & & & & & & & 5.93 & 3.64 & 9.66 \\
\hline Percent American Indian & & & & & & & 2.70 & 2.01 & 3.61 \\
\hline Percent Asian/Pacific Islander & & & & & & & 1.66 & 1.41 & 1.94 \\
\hline
\end{tabular}

$I R R$ Incidence rate ratio, $C I$ Confidence interval, ref. referent

shows that the case fatality ratio remained significantly high in rural counties after adding the percent 2-week increase in deaths and cases. Our analysis shows that percentage of 2week increase in deaths was positively associated with an increase in COVID-19 CFR while the percentage of 2-week increase in cases was not significantly associated.

\section{Spatial Analysis of COVID-19 CFR in Rural Counties}

Figure 5 shows the local distribution of predictors of COVID19 CFR in rural counties. Figure 5a shows the percentage of Black, indigenous, and people of color (BIPOC) and the percentage votes for Trump predict a high fatality ratio in rural counties in southeastern states. The local coefficients for each predictor are presented in Fig. 5b-f. Percentage of the Black population was associated with the clusters of COVID-19

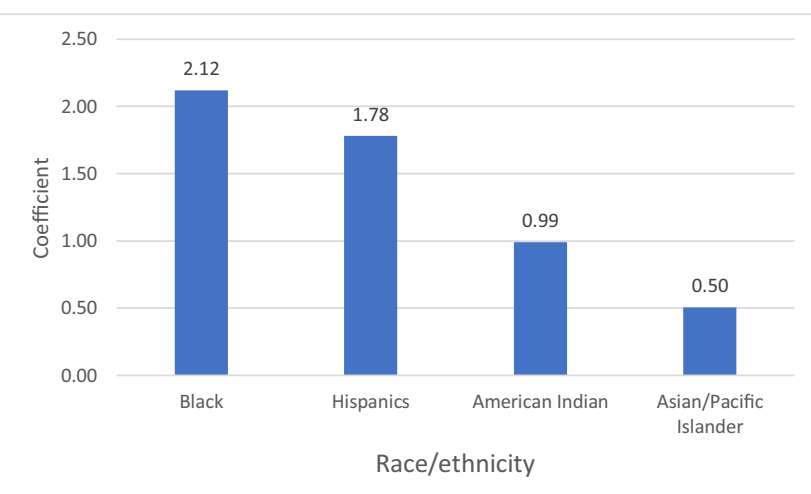

Fig. 2 Racial/ethnic disparity in COVID-19 case fatality ratio in ruralurban counties (adjusted)
CFR in rural counties in Midwest states (e.g., Mexico, Oklahoma, Kansas, Missouri, Illinois, Kentucky, Tennessee, and Indiana). To the west, percent American Indian in Nevada, Utah, Arizona, Colorado, New Mexico, Kansas, the southern part of Nebraska, and Wyoming was associated with the cluster of COVID-19 CFR. Furthermore, toward the east, counties in Illinois, Indiana, Kentucky, Alabama, and Georgia formed a cluster of high CFR, and the percentage of American Indians in the Midwest, Southwest, and Rocky Mountains was associated with COVID-19 CFR.

Percent Hispanics in rural counties was also positively correlated with COVID-19 CFR in most Midwest, Mid-Atlantic, and Northeast regions, including New York, Massachusetts, Maine, Pennsylvania, and Maryland. We also see a cluster of counties where percent Hispanics predict a positive rate in Indiana, Iowa, Illinois, Indiana, Missouri, and Kentucky. Similarly, the share of Asian/Pacific Islander was positively associated with a high fatality ratio in southeastern states, including Florida, Louisiana, Mississippi, Alabama, Georgia, Tennessee, Kentucky, Indiana, Illinois, Michigan, and mostly all states in the extreme Northeast region (Fig. 5e). Figure $5 \mathrm{f}$ shows that the percentage of Trump voters significantly predicts a positive CFR in rural counties in the Midwest region.

Model prediction deviance residual presented in Fig. $5 \mathrm{~g}$ is the difference between the computed and predicted rates. The condition number presented in Fig. 5h shows that there is no multicollinearity among the predictors. Furthermore, the model residual was subjected to a spatial autocorrelation test using the global Moran's $I$ index. The result indicates a good model fit (Moran's $I=0.014$, expected, -0.0004 ), and the prediction was not bias according to the $Z$-score of 1.07 ( $p=0.284)$. 
Table 3 Test of association between racial/ethnic composition, political inclination, and COVID-19 case fatality ratio in rural counties

\begin{tabular}{|c|c|c|c|c|c|c|}
\hline \multirow[t]{3}{*}{ Parameters } & \multicolumn{3}{|c|}{ Model 1} & \multicolumn{3}{|c|}{ Model 2} \\
\hline & \multirow[t]{2}{*}{ IRR } & \multicolumn{2}{|l|}{$95 \% \mathrm{CI}$} & \multirow[t]{2}{*}{ IRR } & \multicolumn{2}{|c|}{$95 \% \mathrm{CI}$} \\
\hline & & Lower & Upper & & Lower & Upper \\
\hline (Intercept) & 6.018 & 5.662 & 6.40 & 5.324 & 3.598 & 7.876 \\
\hline Trump vote (Biden $\left.{ }^{\text {ref. }}\right)$ & & & & 0.864 & 0.386 & 1.934 \\
\hline \multicolumn{7}{|c|}{ Racial/ethnic composition (White ${ }^{\text {ref. }}$ ) } \\
\hline Percent Black & 8.621 & 4.304 & 17.30 & 8.576 & 3.905 & 18.833 \\
\hline Percent Hispanics & 5.867 & 3.101 & 11.10 & 6.155 & 2.99 & 12.67 \\
\hline Percent American Indian & 2.611 & 1.778 & 3.83 & 2.684 & 1.745 & 4.129 \\
\hline Percent Asian/Pacific Islander & 1.357 & 1.111 & 1.66 & 1.255 & 0.828 & 1.904 \\
\hline Median household income & & & & 0.809 & 0.743 & 0.882 \\
\hline Population density & & & & 0.881 & 0.135 & 5.758 \\
\hline
\end{tabular}

IRR Incidence rate ratio, $C I$ Confidence interval, $r e f$. referent

\section{Discussions}

It is apparent that COVID-19 has exacerbated health inequality and reduced life expectancy in the USA $[31,34]$. This study has shown the disparity in COVID-19 case fatality ratio, and it aligns with other studies situated within the social determinant of health framework, as shown in the literature $[17,18,48]$. According to the World Health Organization [49], the social determinant of health suggests that race/ethnicity, power, and location determine health outcomes

Table 4 Sensitivity analysis testing the potential impact of the 2-week increase in deaths and cases on the COVID-19 case fatality ratio

\begin{tabular}{llll}
\hline Parameters & IRR & \multicolumn{2}{l}{$95 \% \mathrm{CI}$} \\
\cline { 3 - 4 } & & Lower & Upper \\
\hline (Intercept) & 5.44 & 5.10 & 5.81 \\
Mostly rural & 1.12 & 1.02 & 1.23 \\
Completely rural & 1.30 & 1.15 & 1.46 \\
Biden vote & 0.93 & 0.85 & 1.02 \\
Trump vote & 1.07 & 0.98 & 1.16 \\
College degree & 0.88 & 0.85 & 0.92 \\
Population density & 1.06 & 1.01 & 1.11 \\
Household income & 0.89 & 0.85 & 0.93 \\
Percent Black & 5.21 & 3.18 & 8.53 \\
Percent Hispanics & 3.94 & 2.50 & 6.20 \\
Percent American Indian & 2.03 & 1.55 & 2.66 \\
Percent Asian/Pacific Islander & 1.48 & 1.27 & 1.71 \\
2-week increase: death & 1.43 & 1.38 & 1.49 \\
2-week increase: cases & 0.82 & 0.78 & 0.85 \\
\hline
\end{tabular}

$I R R$ Incidence rate ratio, $C I$ Confidence interval for an individual or a group of people. This study shows evidence of racial/ethnic disparity in the severity of the pandemic. It further presents how the political inclination may influence the disproportionate distribution of fatal cases of COVID-19.

An apparent rural-urban disparity exists in the distribution of COVID-19 severity. This study observed a mean case fertility rate of $1.79 \%$ for rural counties, higher than the mean of 1.69 at the national level. Overall, cluster analysis indicates variation in the hotspot of COVID-19 CFR. Figure 3 elucidates that rural counties in the panhandle and southern part of Texas, the western part of New Mexico, Louisiana, Mississippi, Alabama, and Georgia, South Carolina, South of Illinois, and North of South Virginia have significant hotspots of COVID-19 CFR compared to some rural counties in Colorado and California. Most of these counties also have a significant proportion of BIPOC, which aligns with another study that indicates that COVID-19 deaths are higher in rural counties with a higher share of Black and Hispanic populations [11]. The significant hotspot could be attributed to some of the determinants of health, such as poor access to medical and socioeconomic resources.

The primary determinant of health inequality among minority groups in the USA is poverty. For example, African Americans have intergenerational poverty, which reflects in their health conditions [50]. In the pre-pandemic, African Americans and Hispanics had the least access to health care in the USA due to their inability to access health insurance principally because of low income [18]. Also, they are more likely to have marginal income above the poverty line than their White counterparts. Recent research has shown that African Americans are likely to have chronic health conditions predisposing them to COVID-19, 
Fig. 3 Geographic distribution of case fatality ratio in rural counties and hotspots
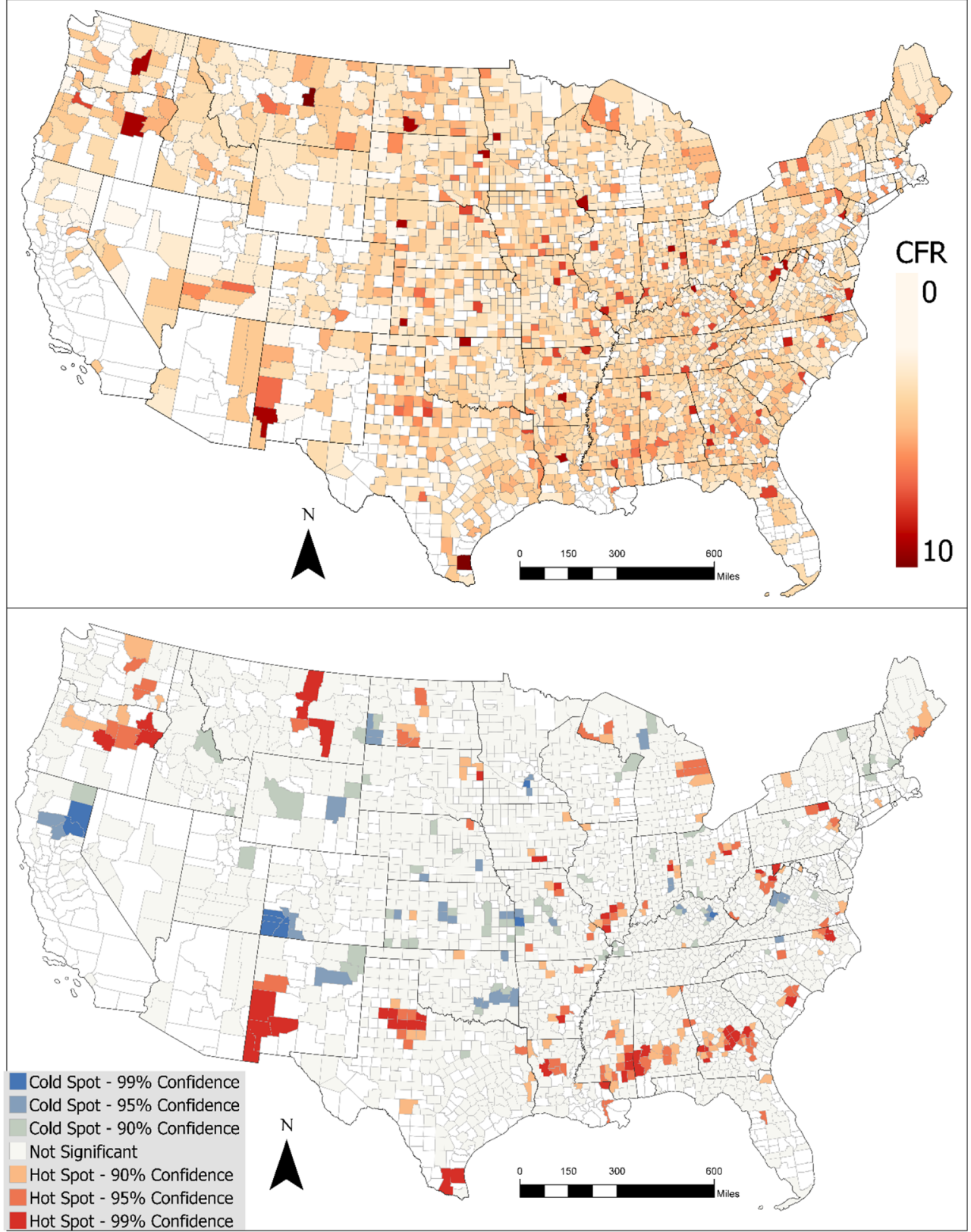

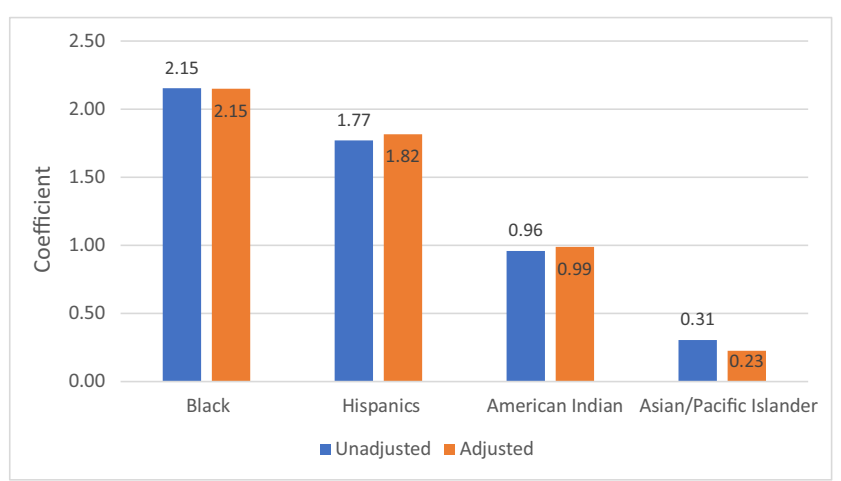

Fig. 4 Racial/ethnic disparity in COVID-19 case fatality ratio in US rural counties (note: the model adjusted for population density, political inclination, and county-level household median income) mostly because of the longtime racial health disparity in health care [50]. Our study further provides evidence of health disparity by race/ethnicity and by location of residence, corroborating studies on racial and ethnic health disparity in the USA.

The global and local statistics results showed that the percentage of Blacks, indigenes, and people of color positively associated with COVID-19 severity in rural counties. The disparity in COVID-19 severity in this study further echoes findings from previous studies on race/ethnic health disparities in the USA. Many factors could account for the observed disparity in COVID-19 severity in the USA, including structural racism, shortage in health care resources [15], and lack of socioeconomic opportunities that can 
Fig. 5 Results of geographically weighted Poisson regression. a Predicted rates. b-e Coefficients of percent Black, indigenous, and people of color in rural counties. $\mathbf{f}$ Coefficient of percent share of Trump 2020 votes. g Deviance residual. $\mathbf{h}$ Condition number

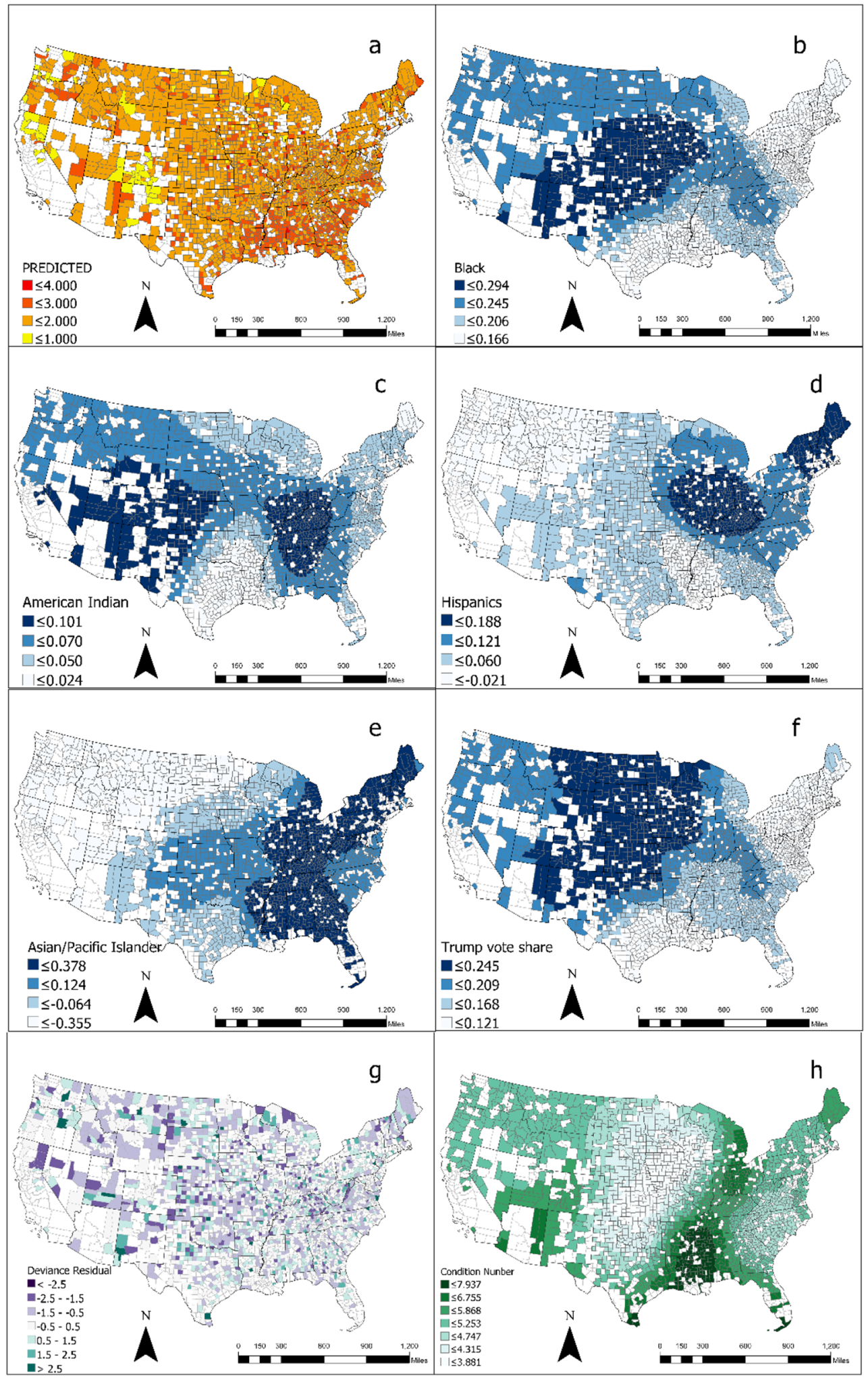

transform the standard of living of racial/ethnic minorities $[48,51]$.

In rural counties alone, the percentage of Blacks, indigenes, and people of color (BIPOC) positively associated with a high case fatality rate. After adjusting for other known determinants of health in rural literature, the association between the percentages of Blacks in rural counties remained significant and unchanged. However, it increased for Hispanics and 
American Indians but reduced for Asian/Pacific Islander. These findings align with previous literature on BIPOC health inequality in rural counties in the USA [10]. Our findings support previous studies and showed that Hispanics are at higher risk of infection and death from coronavirus. In the USA, particularly New York City (NYC) has borne the significant impact of COVID-19 with the highest reported number of cases and deaths in the country. Based on spatial analysis, Cordes and Castro [52] show that percentage of Hispanics and Blacks was positively correlated with the rate of confirmed cases in NYC. Our results also confirm a study that constructed COVID-19 outcome continua in NY state. Holtgrave et al. [53] found a significant disparity in fatality ratio by race/ethnicity and showed that Hispanics have a 3.48fold disparity while Blacks had a 5.38-fold disparity compared to White.

The global analysis showed that the percentage of votes for the Republican presidential candidate had a negative relationship with high case fatality. This study also shows how the political inclination may be associated with the distribution of COVID-19 severity. Based on the evidence from the spatial analysis, there exists a positive link between political inclination and COVID-19 CFR. The spatial analysis provides a more in-depth insight into the association between the dependent variables and predictors not explained in the global regression. COVID-19 CFR was high in rural counties where the Republican presidential candidate had a significant percentage of voters in the Midwest region. The spatial analysis revealed an important insight masked in the conventional regression model. Our study demonstrates the importance of spatial statistics in epidemiology. The potential impact of political inclination on the distribution of COVID-19 CFR could be further explained as an upstream factor with a local effect at the local/ community level. In April 2020, when the CDC updated public health recommendations about the mandatory wearing of face masks in public places, President Trump said, "I don't think that I'm going to be doing it"; wearing a mask will "send the wrong message" to his followers [27]. As a result, many Trump supporters do not support the wearing of masks in public places. According to NBC News [27], "politicians of both parties are clued into the powerful symbolism of the mask, and many Americans take their cues from the president." This statement reinforces findings from this study, which could explain the distribution of COVID-19 case fatality ratio across rural counties.

Geographically focusing on policies that will eliminate the most important determinant of health, poverty, is crucial to placate COVID-19 disparity among racial/ethnic groups in the USA. Our study underscores that improved income would bridge the current health inequality. We found a consistent inverse relationship between county-level median household income and COVID-19 CFR. Research has proposed how and why poverty and inequality can drive inequality in COVID-19 infection and mortality [51, 54, 55]. For example, African Americans have intergenerational poverty, which reflects in their health conditions [50]. In the pre-pandemic, African Americans and Hispanics had the least access to health care in the USA due to their inability to access health insurance principally because of low income [18]. Our study further provides evidence of health disparity by race/ethnicity and by place of residence, corroborating studies on racial and ethnic health disparity in the USA.

The findings from this study must be interpreted with caution based on some methodological limitations, including ecological fallacy. First, we analyzed county-level data, not individual, which prevents us from validly concluding that counties with a high concentration of BIPOC will have a high COVID-19 CFR. Because health data are highly sensitive data, individual-level data (e.g., race/ethnicity, gender, age, place of residence) were not available, which would have benefited this study. Health data are not usually available at the fine granular scale that can support the required, more thorough analysis, especially in minority communities. As submitted by McLaren [48], data paucity is the most significant limitation in finding the sources of disparity in social inequality/inequity research.

The second potential limitation is the use of the case fatality ratio. The use of CFR may lead to conditional CFR estimates since the current COVID-19 is still an ongoing pandemic. The current CFR estimate is likely to be influenced by the lags in report dates for cases and deaths [4]. Hence, the use of CFR remains controversial due to its reliance on testing. Another critical challenge with accurate calculation of the CFR is the denominator: the number of people who are infected with the virus. Notably, asymptomatic cases of COVID-19 and patients with mild symptoms or misdiagnosed individuals could be left out of the denominator, leading to CFR underestimation and overestimation [56]. Our CFR calculation is likely to be skewed due to the inequality in testing. However, it seems plausible to examine the COVID-19 CFR after millions of testing have already been conducted in the USA in 2020 to show the first year fatality rate from COVID-19. Testing in the USA has significantly increased [57], which, to some extent, gives credence to the CFR calculation at this time as conditional estimates. This current estimation can serve as a reference for future estimations and at the end of the pandemic.

Despite these limitations, this study contributes to the epidemiological understanding of the impact of COVID19 on US rural communities. The findings have significant implications for public health intervention, rural or mostly in rural counties with the elevated percentage of Black, indigenes, and people of color who face the major brunt of the current pandemic and other structural 
disparity. Compared to global econometric methods used in public health research, we combined global and local analysis to examine geographic (spatial) and racial/ethnic heterogeneity in COVID-19 CFR in the USA. Since human behaviors vary across the idiosyncratic sociophysical environment, public health practitioners are interested in tools that could easily identify variability across geographic settings such as the rural-urban continuum. Public health interventions and health policies cannot be a "fit it all," and policymakers must consider the specific local context when making decisions that can translate to effective positive outcomes. Hence, it is crucial to adjust public policy needs geographically and apply only in areas considered the most priority.

\section{Conclusion}

Health disparity is noticeable in marginalized communities, particularly during any disaster, such as the current COVID19 pandemic. This study showed that rural counties have a COVID-19 case fatality ratio higher than the national rate. Black, indigenous, and people of color are more likely to be impacted. Political inclination and the existing social determinant of health (e.g., inequality) explain the geographic disparity of the COVID-19 case fatality ratio in rural counties. Furthermore, minority populations in rural counties are disproportionately more likely to be infected and die from the disease. Reversing the US's current inequalities, particularly in rural counties, should be the highest priority in the post-pandemic era. Policies should prioritize rural health and adequate distribution of health care resources to meet the need of the minority populations, particularly in rural counties identified in this study. Lastly, improving the socioeconomic position of rural minorities can reduce the effect of structural determinants that expose them to various health risks.

Supplementary Information The online version contains supplementary material available at https://doi.org/10.1007/s40615-021-01006-7.

Code Availability See supplementary file.

Authors' Contributions All authors contributed significantly.

Data Availability Data are publicly available online from DOI 10.17605/ OSF.IO/WD2N6.

\section{Declarations}

Conflict of Interest The authors declare no competing interests.

Disclaimer All views are of the authors.

\section{References}

1. Cheng VC, Lau SK, Woo PC, Yuen KY. Severe acute respiratory syndrome coronavirus as an agent of emerging and reemerging infection. Clinical microbiology reviews. Am Soc Microbiol. 2007;20:660-94.

2. Onder G, Rezza G, Brusaferro S. Case-fatality rate and characteristics of patients dying in relation to COVID-19 in Italy. JAMA. 2020:323, 1775-1326 [cited 2021 Jan 20] Available from: https:// doi.org/10.1001/jama.2020.4683, Case-Fatality Rate and Characteristics of Patients Dying in Relation to COVID-19 in Italy.

3. World Health Organization. Estimating mortality from COVID-19: scientific brief. 2020 [cited 2021 Jan 23]. Available from: https:// www.who.int/news-room/commentaries/detail/estimatingmortality-from-covid-19

4. Spychalski P, Błażyńska-Spychalska A, Kobiela J. Estimating case fatality rates of COVID-19. Lancet Infect Dis. 2020;20:774-5 [cited 2021 Jan 20] Available from: https://www.ncbi.nlm.nih.gov/ pmc/articles/PMC7270730/.

5. Porta M. A dictionary of epidemiology: Oxford university press; 2014.

6. Centers for Disease Control and Prevention. Coronavirus Disease 2019 (COVID-19) situation summary. Centers Dis Control Prev. 2020; [cited 2020 Mar 26]. Available from: https://www.cdc.gov/ coronavirus/2019-ncov/cases-updates/summary.html.

7. Holshue ML, DeBolt C, Lindquist S, Lofy KH, Wiesman J, Bruce $\mathrm{H}$, et al. First case of 2019 novel coronavirus in the United States. New England Journal of Medicine. Mass Medical Soc. 2020; First Case of 2019 Novel Coronavirus in the United States.

8. Yang Y, Peng F, Wang R, Guan K, Jiang T, Xu G, et al. The deadly coronaviruses: the 2003 SARS pandemic and the 2020 novel coronavirus epidemic in China. Journal of autoimmunity. Elsevier; 2020;102434.

9. Lovelace BJ. The coronavirus may be deadlier than the 1918 flu: here's how it stacks up to other pandemics: CNBC; 2020. [cited 2020 Apr 15]. Available from: https://www.cnbc.com/2020/03/26/ coronavirus-may-be-deadlier-than-1918-flu-heres-how-it-stacksup-to-other-pandemics.html

10. Worldometer. Coronavirus: live update of reported cases and deaths from COVID-19 virus pandemic [Internet]. 2020 [cited 2020 Apr 15]. Available from: https://www.worldometers.info/ coronavirus/

11. Cheng KJG, Sun Y, Monnat SM. COVID-19 death rates are higher in rural counties with larger shares of Blacks and Hispanics. J Rural Health. Wiley Online Library. 2020;36:602-8.

12. Peters DJ. Community susceptibility and resiliency to COVID-19 across the rural-urban continuum in the United States. The Journal of Rural Health. Wiley Online Library. 2020;36:446-56.

13. Cohen PN. The COVID-19 epidemic in rural U.S. Counties. European J Env Publi. 2020;4:em0050 Veritas Publications LTD; [cited 2020 Dec 21];. Available from: https://www.ejeph.com/ article/the-covid-19-epidemic-in-rural-us-counties-8331.

14. Norris K, Gonzalez C. COVID-19, health disparities and the US election. EClinicalMedicine. 2020; [cited 2020 Dec 22];28. Available from: https://www.thelancet.com/journals/eclinm/ article/PIIS2589-5370(20)30361-8/abstract.

15. Barreto T, Jetty A, Eden AR, Petterson S, Bazemore A, Peterson LE. Distribution of physician specialties by rurality. J Rural Health. 2020 [cited 2020 Dec 24]; Available from. https://doi.org/10.1111/ jrh.12548.

16. Singh GK, Daus GP, Allender M, Ramey CT, Martin EK, Perry C, et al. Social determinants of health in the United States: addressing major health inequality trends for the nation, 1935-2016. Int J MCH AIDS. 2017;6:139-64 [cited 2020 Dec 20];. Available from: https://www.ncbi.nlm.nih.gov/pmc/articles/PMC5777389/. 
17. Sood L, Sood V. Being African American and rural: a double jeopardy from Covid-19. J Rural Health. 2020.

18. Snowden LR, Graaf G. COVID-19, social determinants past, present, and future, and African Americans' health. J Racial Ethnic Health Disparities. 2020 [cited 2020 Dec 29]; Available from;8: 12-20. https://doi.org/10.1007/s40615-020-00923-3.

19. Zhang $\mathrm{CH}$, Schwartz GG. Spatial disparities in coronavirus incidence and mortality in the United States: an ecological analysis as of May 2020. J Rural Health. 2020;36:433-45.[cited 2020 Dec 20] Available from:. https://doi.org/10.1111/jrh.12476.

20. Seligman B, Ferranna M, Bloom DE. Social determinants of mortality from COVID-19: a simulation study using NHANES. PLOS Med. 2021;18:e1003490 Public Library of Science; [cited 2021 Jan 21]. Available from: https://journals.plos.org/plosmedicine/ article?id=10.1371/journal.pmed. 1003490 .

21. Iyanda AE, Adeleke R, Lu Y, Osayomi T, Adaralegbe A, Lasode $\mathrm{M}$, et al. A retrospective cross-national examination of COVID-19 outbreak in 175 countries: a multiscale geographically weighted regression analysis (January 11-June 28, 2020). J Infect Public Health. 2020;13:1438-45 [cited 2020 Oct 12]Available from: http://www.sciencedirect.com/science/article/pii/ S1876034120305724.

22. Sorci G, Faivre B, Morand S. Explaining among-country variation in COVID-19 case fatality rate. Scientific Reports. 2020:10, 18909 Nature Publishing Group; [cited 2021 January 21y 21]. Available from: https://www.nature.com/articles/s41598-020-75848-2.

23. Sun F, Matthews SA, Yang T-C, Hu M-H. A spatial analysis of the COVID-19 period prevalence in U.S. counties through June 28, 2020: where geography matters? Ann Epidemiol. 2020;52:5459.e1 10:18909Available from: http://www.sciencedirect.com/ science/article/pii/S104727972030274X.

24. Andersen LM, Harden SR, Sugg MM, Runkle JD, Lundquist TE. Analyzing the spatial determinants of local Covid-19 transmission in the United States. Sci Total Environ. 2021;754:142396 [cited 2021 Jan 21]Available from: http://www.sciencedirect.com/ science/article/pii/S0048969720359258.

25. Khan JR, Awan N, Islam MM, Muurlink O. Healthcare capacity, health expenditure, and civil society as predictors of COVID-19 case fatalities: a global analysis. Front Public Health. 2020;8 [cited 2021 Jan 26];. Available from: https://www.frontiersin.org/articles/ 10.3389/fpubh.2020.00347/full?report=reader.

26. Ge Y, Sun S, Shen Y. Estimation of case-fatality rate in COVID-19 patients with hypertension and diabetes mellitus in the New York state: a preliminary report. Epidemiol Infect. 2021;149 [cited 2021 January 21y 21]. Cambridge University Press; Available from: https://www.cambridge.org/core/journals/epidemiology-andinfection/article/estimation-of-casefatality-rate-in-covid19-patientswith-hypertension-and-diabetes-mellitus-in-the-new-york-state-apreliminary-report/9EB6172951AFC01E14338554513E157A.

27. NBC News. Trump tells allies his wearing a mask would "send the wrong message," make him look ridiculous [Internet]: NBC News; 2020. [cited 2020 Dec 23]. Available from: https://www.nbcnews. com/politics/donald-trump/trump-tells-allies-his-wearing-maskwould-send-wrong-message-n1202001

28. Do CW. Republicans or Democrats benefit from mail-in voting? It turns out, neither. Science. 2020; [cited 2021 Jan 20]. Available from: https://www.sciencemag.org/news/2020/08/do-republicansor-democrats-benefit-mail-voting-it-turns-out-neither.

29. Li R, Richmond P, Roehner BM. Effect of population density on epidemics. Physica A: Statistical Mechanics and its Applications. Elsevier. 2018;510:713-24.

30. Goujon A, Natale F, Ghio D, Conte A, Dijkstra L. Age, gender, and territory of COVID-19 infections and fatalities. EUR; 2020.

31. van Dorn A, Cooney RE, Sabin ML. COVID-19 exacerbating inequalities in the US. Lancet. 2020;395:1243-4 [cited 2020 Dec 20].
Available from: https://www.ncbi.nlm.nih.gov/pmc/articles/ PMC7162639/.

32. Yancy CW. COVID-19 and African Americans. JAMA. 2020;323: 1891-2 [cited 2020 Dec 20]. Available from, COVID-19 and African Americans. https://doi.org/10.1001/jama.2020.6548.

33. Singh GK, Siahpush M. Widening rural-urban disparities in life expectancy, US, 1969-2009. American journal of preventive medicine. Elsevier. 2014;46:e19-29.

34. Arias E, Tejada-Vera B, Ahmad F. Provisional Life Expectancy Estimates for January through June 2020. Report No. 10. February 2021. Available from: https://www.cdc.gov/nchs/data/ vsrr/VSRR10-508.pdf

35. Hooper MW, Nápoles AM, Pérez-Stable EJ. COVID-19 and racial/ ethnic disparities. Jama. 2020.

36. Macias Gil R, Marcelin JR, Zuniga-Blanco B, Marquez C, Mathew T, Piggott DA. COVID-19 pandemic: disparate health impact on the Hispanic/Latinx population in the United States. J Infect Dis. 2020;222:1592-5.

37. Renelus BD, Khoury NC, Chandrasekaran K, Bekele E, Briggs WM, Ivanov A, et al. Racial disparities in covid-19 hospitalization and in-hospital mortality at the height of the New York City pandemic. J Racial Ethnic Health Disparities. 2020:1-7.

38. Rodriguez-Diaz CE, Guilamo-Ramos V, Mena L, Hall E, Honermann B, Crowley JS, et al. Risk for COVID-19 infection and death among Latinos in the United States: examining heterogeneity in transmission dynamics. Ann Epidemiol. 2020;52:46-53.

39. New York Times. nytimes/covid-19-data [Internet]. The New York Times; 2020 [cited 2020 December 21er 21]. Available from: https://github.com/nytimes/covid-19-data

40. US Census Bureau. County population by characteristics: 20102019 [Internet]. The United States Census Bureau. [cited 2020 Dec 21]. Available from: https://www.census.gov/data/tables/ time-series/demo/popest/2010s-counties-detail.html

41. Cohen PN. Coronavirus: OSF; 2020. [cited 2021 Jan 20]; Available from: https://osf.io/wd2n6/

42. Vestal AJ, Briz A, Choi A, Jin B, McGill A, Mihalik L. Live 2020 election results: Presidency, Senate and House. 2020 [cited 2021 Jan 20]. Available from: https://www.politico.com/2020-election/ results/

43. Longjian L, Katsumi I, Yukio Y. Changes in stroke mortality rates for 1950 to 1997. Stroke. 32:1745-9American Heart Association; 2001 [cited 2021 January 23y 23]. Available from. https://doi.org/ 10.1161/01.STR.32.8.1745.

44. Fenta SM, Fenta HM, Ayenew GM. The best statistical model to estimate predictors of under-five mortality in Ethiopia. J Big Data. 2020;7:63. https://doi.org/10.1186/s40537-020-00339-0 [cited 2021 Jan 23] Available from: The best statistical model to estimate predictors of under-five mortality in Ethiopia.

45. IBM Corp. Released 2011. IBM SPSS Statistics for Windows, Version 20.0. Armonk, NY: IBM Corp; 2011. [cited 2018 Sep 13]. Available from: http://www-01.ibm.com/support/docview. wss?uid=swg21476197

46. Brunsdon C, Fotheringham AS. Charlton ME. Geographically weighted regression: a method for exploring spatial nonstationarity. Geographical Analysis. 1996;28:281-98. [cited 2018 Nov 8];Available from. https://doi.org/10.1111/j.1538-4632.1996. tb00936.x.

47. ESRI. ArcGIS Pro |2D, 3D \& 4D GIS Mapping Software [Internet]. 2018 [cited 2020 Dec 24]. Available from: https:// www.esri.com/en-us/arcgis/products/arcgis-pro/overview

48. McLaren J. Racial disparity in COVID-19 deaths: seeking economic roots with census data. NBER working paper. 2020.

49. World Health Organization. Commission on Social Determinants of Health-CSDH. 2008; 
50. Gravlee CC. Systemic racism, chronic health inequities, and COVID-19: a syndemic in the making? Wiley-Blackwell: American Journal of Human Biology; 2020.

51. Abedi V, Olulana O, Avula V, Chaudhary D, Khan A, Shahjouei S, et al. Racial, economic, and health inequality and COVID-19 infection in the United States. J Racial Ethnic Health Disparities. 2020: $1-11$.

52. Cordes J, Castro MC. Spatial analysis of COVID-19 clusters and contextual factors in New York City. Spatial Spatio Temporal Epidemiol. 2020;34:100355 [cited 2020 Dec 23]. Available from: http://www.sciencedirect.com/science/article/pii/ S1877584520300332.

53. Holtgrave DR, Barranco MA, Tesoriero JM, Blog DS, Rosenberg ES. Assessing racial and ethnic disparities using a COVID-19 outcomes continuum for New York State. Ann Epidemiol. 2020;48:914 [cited 2020 Dec 23] Available from: http://www.sciencedirect. com/science/article/pii/S1047279720302076.
54. Patel JA, Nielsen FBH, Badiani AA, Assi S, Unadkat VA, Patel B, et al. Poverty, inequality and COVID-19: the forgotten vulnerable. Public Health. 2020;183:110.

55. Ahmed F, Ahmed N, Pissarides C, Stiglitz J. Why inequality could spread COVID-19. Lancet Public Health. 2020;5:e240.

56. Rajgor DD, Lee MH, Archuleta S, Bagdasarian N, Quek SC. The many estimates of the COVID-19 case fatality rate. Lancet Infect Dis. 2020;20:776-7.

57. Martell A, Parker N. The U.S. has more COVID-19 testing than most. So why is it falling so short? Reuters. 2020; 277 [cited 2021 January 20y 20]; Available from: https://www.reuters.com/article/ us-health-coronavirus-usa-testing-insigh-idUSKCN24S19H.

Publisher's Note Springer Nature remains neutral with regard to jurisdictional claims in published maps and institutional affiliations. 